\title{
'Treat to target': moving targets from hypertension, hyperlipidaemia and diabetes to rheumatoid arthritis
}

\author{
Dan Atar, 1,2 Kåre Inge Birkeland, ${ }^{2,3}$ Till Uhlig ${ }^{4}$
}

The principle of 'treat to target' embraces an indispensable approach to the prevention of some of the most prevalent diseases: diabetes, arterial hypertension and coronary heart disease. These highly ubiquitous disorders account for most deaths and disabilities worldwide. Preventive treatment is thus a matter of paramount priority.

How did 'treat to target' come about? The rationale for this therapeutic paradigm is based on a comprehensive evidence base that is-fair to say-unique to cardiovascular medicine. Few areas in contemporary medicine have investigated hundreds of thousands of patients with a given condition just to scrutinise one particular therapeutic intervention, such as the lowering of blood pressure with a given agent, or such as low-density lipoprotein (LDL)-cholesterol lowering by a statin.

What do we mean by 'comprehensive evidence base'? Clinical trials demonstrate that to reduce cardiovascular events in individuals with hypertension, blood pressure lowering (eg, to $<150 / 100$ $\mathrm{mm} \mathrm{Hg}$ ) is effective, and more intensive blood pressure lowering (eg, to $<140 / 90$ $\mathrm{mm} \mathrm{Hg}$ ) is more effective. ${ }^{1}$ Likewise, to reduce cardiovascular events in patients with established ischaemic heart disease through lipid lowering, a statin is more effective than placebo, aggressive statin therapy is more effective than lenient statin therapy, and in patients receiving aggressive statin therapy the

\footnotetext{
'Department of Cardiology, Oslo University Hospital, Aker and Ullevål, Oslo, Norway

${ }^{2}$ Faculty of Medicine, University of Oslo, Oslo, Norway

${ }^{3}$ Department of Endocrinology, Oslo University Hospital, Oslo, Norway

${ }^{4}$ Department of Rheumatology, Diakonhjemmet Hospital, Oslo, Norway
}

Correspondence to Professor Dan Atar, Department of Cardiology, Division of Medicine B, Oslo University Hospital, Kirkeveien 166, Oslo 0407, Norway; dan.atar@medisin.uio.no lowest risk is associated with the lowest LDL-cholesterol levels. ${ }^{2}$ This preventive treatment strategy aiming at lowering LDL-cholesterol has been coined as the concept of 'the lower the better'. Incidentally, the aphorism 'the lower the better' is just a linguistic corollary to the dictum 'treat to target'. Taken together, this evidence base essentially teaches us that treating patients to goal confers the best outcome.

Is the 'treat to target' principle reflected in current cardiovascular treatment guidelines? In fact, it is firmly reinforced in cardiovascular practice guidelines and, accordingly, is rigorously employed in cardiovascular medicine. Guidelines for the prevention of cardiovascular disease are consistent in their recommendations regarding antihypertensive treatment, statin therapy andmost importantly-treatment targets. For instance, the European Society of Cardiology guidelines on cardiovascular disease prevention recommend a total cholesterol goal of less than 4.5 (preferably <4.0) and a LDL-cholesterol goal of less than 2.5 (preferably <2.0). ${ }^{3}$ These targets by and large confer the highest probability of survival in patients at risk of cardiovascular events. In conclusion, comprehensive scrutiny and scientific evidence has led to the stringent implementation of 'treat to target' in cardiovascular medicine.

In diabetes, treatment to glucose level targets has been the leading principle as long as glucose measurements have been available, and some of the experiences from diabetes treatment may be worth considering when discussing the 'treat to target' principle in rheumatology. First, two major technical developments have boosted treatment practice in diabetology: The availability of self-measurement of blood glucose to patients and the possibility to evaluate average blood glucose during a period of 6-8 (10) weeks through measuring haemoglobin $A_{1 c}$. Second, it is important to realise that the targets are different whether the objective is to prevent acute symptoms caused by hyperglycaemia or if the aim is to prevent diabetic late complications. Based on these measurements, national and international organisations have established targets for glucose control, with scientific basis mainly in two pivotal clinical trials in type $1^{4}$ and type $2^{5}$ diabetes, which showed a reduction in diabetic late complications with good glycaemic control. The majority of stakeholders have recommended a target for haemoglobin $\mathrm{A}_{1 \mathrm{c}}$ of less than $7 \%$ and corresponding self-measured plasma glucose measurements that correspond to this to obtain a glycaemic control that reduces the risk of diabetic late complications. However, these targets have been under continued debate, and for example a recent joint task force of the European Society of Cardiology and the European Association for the Study of Diabetes recommended a target for haemoglobin $A_{1 c}$ of less than $6.5 \%$ in its 2007 clinical guidelines. ${ }^{6}$ A third important point is the obvious challenge to establish a close association, and preferably causal linkage, between the targets set and the complications we want to prevent. Although this is partly established for blood glucose/haemoglobin $\mathrm{A}_{1 \mathrm{c}}$ and diabetic late complications, other important determinants may interact, such as blood pressure, lipids and a hypercoaguable state. Furthermore, a narrow focus on one target (eg, blood glucose) may result in the neglect of other consequences (eg, risk of hypoglycaemia) with untoward effects. One example of this may be the recently published ACCORD study, which was stopped prematurely by the drug supervision board due to an increase in mortality in intensively treated patients, even though the primary endpoint of the study (a composite of cardiovascular disease) tended to be beneficially influenced by the intensive treatment. ${ }^{7}$ Another very recent study demonstrated that both low and high mean haemoglobin $A_{1 c}$ values in individuals with diabetes are associated with increased all-cause mortality and cardiac events, consistent with a U-shaped curve of risk association. ${ }^{8}$

In rheumatology, the remission of disease activity is now an obvious goal when the rheumatologist treats a patient with rheumatoid arthritis (RA). This ambition in targeting inflammation is currently possible because during the past decade we have gained access to drugs that effectively reduce disease 
activity, improve patient reported outcomes and also prevent structural bone damage.

A task force of rheumatologists has now formulated 10 recommendations for the treatment of $\mathrm{RA},{ }^{9}$ and a literature review has summarised the benefit of target-oriented approaches. ${ }^{10}$ Even though clinical remission is the primary target we want to hit, a level of low disease activity may be an alternative goal to pursue. From trials evaluating the efficacy of biological drugs we do know that for a large portion of patients the ultimate goal of disease remission cannot yet be reached. Nevertheless, we now have a benchmark in rheumatology, a goal to pursue just like in cardiology and diabetology.

Inflammation is the process to interfere with and abrogate or suppress. Inflammation is thus recommended to be monitored by a regular assessment of disease activity. One of the recommendations is to monitor as frequently as every month in patients with high disease activity and as frequently as every third months in patients who have not achieved the target set. ${ }^{9}$ For this purpose, just as cardiologists and diabetologists follow blood pressure and lipid or blood glucose levels, rheumatologists monitor disease activity in RA. Unlike following one biomarker in RA, a composite index, for example the disease activity score in 28 joints (DAS28) ${ }^{11}$ or the clinical disease activity index (CDAI), ${ }^{12}$ is used to obtain information on disease activity. These indices also incorporate the patient perspective as the patient's assessment of disease impact is included. While the rationale for the regular monitoring of disease activity is clear, it seems far more difficult to quantify the frequency of such assessments, and there is very limited level of evidence (level IV) to support firm recommendations. ${ }^{9}$ As a consequence, this recommendation on the frequency of monitoring is also one of those with the lowest agreement among members of the task force, and more research is needed.

The ninth of the recommendations ${ }^{9}$ reminds us that the level of disease activity to aim for will be influenced by patient-related factors such as comorbidities and the risks of toxicity inherent to a drug. This indicates that treatment targets need to be adapted to the individual patient situation. For example, in a patient with RA in whom the forefeet or ankles are affected, these joints are not aggregated into the DAS28, and the DAS28 may then not be the most adequate measure of disease activity. Obviously, comorbidities and experienced drug toxicity may also lead the treating rheumatologist to deviate from pure numbers of disease activity given as treatment targets.

There is now a benchmark (DAS28 $<2.6)$ for treating disease activity in RA, and useful recommendations support the 'treat to target' principle. The rheumatology community needs to work with the implementation of these recommendations persistently to pursue improved patient outcomes.

\section{Competing interests None.}

Provenance and peer review Commissioned; externally peer reviewed.

\section{Accepted 2 February 2010}

Ann Rheum Dis 2010;69:629-30.

doi:10.1136/ard.2010.128462

\section{REFERENCES}

1. Staessen JA, Wang JG, Thijs L. Cardiovascular protection and blood pressure reduction: a metaanalysis. Lancet 2001;358:1305-15.

2. Cannon CP. The IDEAL cholesterol: lower is better. JAMA 2005;294:2492-4.

3. Graham I, Atar D, Borch-Johnsen K, et al. European guidelines on cardiovascular disease prevention in clinical practice: executive summary. Eur Heart J 2007; 28:2375-414

4. The Diabetes Control and Complications Trial Research Group. The effect of intensive treatment of diabetes on the development and progression of long-term complications in insulindependent diabetes mellitus. $N$ Engl J Med 1993;329:977-86.

5. UK Prospective Diabetes Study Group Intensive blood glucose control with sulphonylureas or insulin compared with conventional treatment and risk of complications in patients with type 2 diabetes (UKPDS 33). Lancet 1998;352:837-53.

6. Rydén L, Standl E, Bartnik M, et al. Guidelines on diabetes, pre-diabetes, and cardiovascular diseases: executive summary. The task force on diabetes and cardiovascular diseases of the European Society of Cardiology (ESC) and the European Association for the Study of Diabetes (EASD). Eur Heart J 2007;28:88-136.

7. Gerstein HC, Miller ME, Byington RP, et al. Effects of intensive glucose lowering in type 2 diabetes. N Engl J Med 2008;358:2545-59.

8. Currie CJ, Peters JR, Tynan A, et al. Survival as a function of $\mathrm{HbA}(1 \mathrm{c})$ in people with type 2 diabetes: a retrospective cohort study. Lancet 2010;375:481-9.

9. Smolen JS, Aletaha D, Bijlsma JWJ, et al. Treating rheumatoid arthritis to target: recommendations of an international task force. Ann Rheum Dis 2010;69:631-7.

10. Schoels M, Knevel R, Aletaha D, et al. Evidence for treating rheumatoid arthritis to target: results of a systematic literature research. Ann Rheum Dis 2010;69:638-42.

11. Prevoo ML, van 't Hof MA, Kuper HH, et al. Modified disease activity scores that include twenty-eight-joint counts. Development and validation in a prospective longitudinal study of patients with rheumatoid arthritis. Arthritis Rheum 1995; 38:44-8

12. Aletaha D, Smolen JS. The Simplified Disease Activity Index (SDAl) and Clinical Disease Activity Index (CDAl) to monitor patients in standard clinical care. Best Pract Res Clin Rheumatol 2007;21:663-75. 